\title{
Diffusion Tensor Imaging
}

National Cancer Institute

\section{Source}

National Cancer Institute. Diffusion Tensor Imaging. NCI Thesaurus. Code C64862.

A type of diffusion-weighted magnetic resonance imaging (DW-MRI) that maps the diffusion of water in three dimensions, the principal purpose of which is to image the white matter of the brain, specifically measuring the anisotropy, location, and orientation of the neural tracts, which can demonstrate microstructural changes or differences with neuropathology and treatment. 